This article was published in Crystal Growth and Design, 16(8), 4285-4291, 2016

http://dx.doi.org/10.1021/acs.cgd.6b00334

\title{
The Finding of Nondissolving Lysozyme Crystals and Its Significance for the Study of Hard-to-Crystallize Biological Macromolecules
}

\author{
Cecília Ferreira, ${ }^{\dagger}$ Fernando A. Rocha, ${ }^{\dagger}$ Ana M. Damas, ${ }^{\ddagger}$ and Pedro M. Martins ${ }^{*}, \dagger, \ddagger$

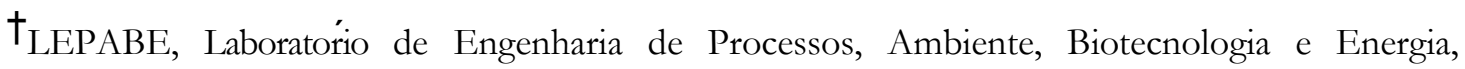 \\ Departamento de Engenharia Química, Faculdade de Engenharia da Universidade do Porto, Rua Dr. \\ Roberto Frias, 4200-465 Porto, Portugal
}

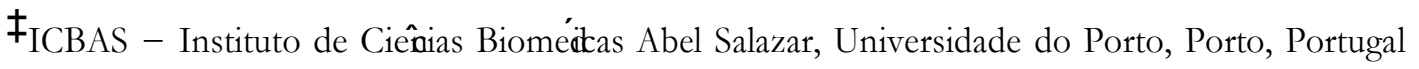

\begin{abstract}
Major discoveries in structural biology depend on obtaining well-diffracting macromolecular crystals. This necessity has motivated many fundamental studies on protein crystallization using lysozyme as a model system. In the present contribution, we report the unprecedented observation of lysozyme crystals that stop dissolving under undersaturated conditions imposed to sub-microliter crystallization drops at mild temperatures. Subsequent growth of the same crystals is apparently undisturbed after the drops are cooled below the saturation temperature. The succession of heating/cooling cycles only partially recovers crystal dissolution while crystal growth becomes gradually slower. Ultimately, increasing and decreasing the temperature between 10 and $37^{\circ} \mathrm{C}$ has no visible effect on the size of the crystals. We ascribe this phenomenon to the partial denaturation of the soluble protein in the drop as evidenced by the decreasing glycoside hydrolase activity of lysozyme over the incubation time. The disturbances in the phase transition processes are explained as the result of the changed chemical potential due to different folding states. In a time when the high-hanging fruits in structural biology have to be picked, the present findings call attention to interfacial phenomena as an important, though often imperceptible, aspect that affects protein stability and justifies further optimization of current crystallization methods.
\end{abstract}

\section{Introduction}

On the occasion of the centennial of X-ray crystallography, a number of thematic reviews placed protein crystallization in a historical context that precedes and ushers in some of the major advances in structural biology over the years. ${ }^{1-3}$ On the other hand, recent reflections and perspectives cite the crystallization of integral membrane proteins, ${ }^{4}$ intrinsically disordered proteins, ${ }^{5}$ macromolecular assemblies, ${ }^{6}$ and nucleoproteic complexes ${ }^{7}$ as the main challenges currently faced by protein crystal growers. Having persisted over the advent of automated 8 and miniaturization 9 methods, recalcitrant proteins pose difficult problems that are hardly solvable by adopting traditional trial-and-error strategies. An alternative biophysical approach relies on the use of 
technological tools rationally designed to control the nucleation step and increase the success rates of crystallization. ${ }^{10}$ Some of the examples already adopted in structural genomic projects involve novel nucleants ${ }^{11}$ and seeding policies, ${ }^{12}$ or crystallization in gel media. ${ }^{13}$ Past contributions from our group showed the beneficial effects on the formation and quality of protein crystals of ultrasonic irradiation, ${ }^{14}$ small temperature oscillations, ${ }^{15}$ and controlled supersaturation build-up profiles. ${ }^{16}$ In turn, the biochemical approach actuates upon the phases that precede nucleation through the development of new ways to stabilize the macromolecular conformation. The success of this strategy is illustrated by the fast increasing number of crystallographic structures involving antibody-protein complexes, ${ }^{5}$ fusion proteins, ${ }^{17}$ and mutated/truncated proteins. ${ }^{18}$ Indeed, the protein itself is confirmed to be the most influential variable in the crystallization process. ${ }^{19-21}$

In the present study, the importance of conformational stability is shown to justify further optimization of current crystallization protocols. Somewhat ironically, this conclusion stems from the observation of the dissolution (rather than crystallization) of hen egg-white lysozyme (HEWL), a stable protein known to readily produce crystals. The nucleation, crystal growth, and phase diagrams of HEWL have been extensively characterized at different temperatures, $\mathrm{pHs}$, and salt concentrations. $^{22-25}$ Comprehensibly from the crystallographer point of view, the dissolution of protein crystals has been much less investigated ${ }^{26}$ in spite of the promising applications envisaged in the controlled delivery of biopharmaceuticals ${ }^{26,27}$ and in industry. ${ }^{28}$ In principle, crystal dissolution is a purely diffusive process driven by the difference in the chemical potential between the undersaturated solution and the crystal lattice. ${ }^{29,30}$ This prompted us to study the dissolution of lysozyme crystals as a probe to monitor variations in the thermodynamic activity in protein solutions caused by, for example, macromolecular crowders. ${ }^{31-33}$ Because lysozyme is a glycoside hydrolase enzyme, the choice of this system allows establishing the relationship between the thermodynamic and the catalytic activities. To our surprise, dissolution rate and enzymatic activity measurements suggest that the structural integrity of the protein is significantly affected under typical crystallization conditions involving no macromolecular additives or denaturants whatsoever.

\section{Experimental Section}

Protein Preparation. HEWL powder obtained from Merck KGaA (Darmstadt, Germany) was dissolved in $0.2 \mathrm{M}$ sodium acetate buffer, $\mathrm{pH} 4.7$, containing 3\% (w/V) sodium chloride. The solutions were prepared at $37{ }^{\circ} \mathrm{C}$ to a final concentration of $25 \mathrm{mg} / \mathrm{mL}$ HEWL and filtered through sterile $0.22 \mu \mathrm{m}$ pore size filters (Millipore Millex-GV).

Crystal Growth/Dissolution. The growth and dissolution of HEWL crystals were observed using a calibrated Nikon SMZ1500 stereomicroscope equipped with a Nikon DS-Ri1 digital camera and Nis-Elements BR 3.1 software. ${ }^{34,35}$ Sub-microliter drops of freshly prepared HEWL solution were placed in a jacketed glass growth cell and covered with $1.0 \mathrm{~mL}$ thermostatized paraffin oil to avoid evaporation. The temperature in the growth cell was decreased at a constant cooling rate from 37.0 to $10.0^{\circ} \mathrm{C}$ over a period of $81 \mathrm{~min}$, after which the temperature was kept constant at $10{ }^{\circ} \mathrm{C}$ during $180 \mathrm{~min}$ to promote the formation and growth of HEWL crystals. To complete the crystallization/dissolution cycle, the temperature was increased from 10.0 to $37.0^{\circ} \mathrm{C}$ over a period 
of $81 \mathrm{~min}$ and kept at $37.0^{\circ} \mathrm{C}$ during the following period of $900 \mathrm{~min}$. The crystallization drops were subject to a succession of six temperature cycles. The projected area of selected HEWL crystals was periodically measured and converted into the equivalent diameter of a circle $(\bar{L})$. The instantaneous rates of crystal growth $\left(R_{\mathrm{G}}\right)$ and dissolution $\left(R_{\mathrm{D}}\right)$ were determined by calculating the slope of linear portion of the $\overline{L v}$ s time curve. After a variable number of cycles, complete dissolution of the HEWL crystal was not possible to achieve at the end of the 900 min dissolution periods.

Enzymatic Assay. The variation of the enzymatic activity of HEWL in 0.7 and $0.4 \mu \mathrm{L}$ crystallization drops was followed during the above-described crystal growth/dissolution experiments. The volume of the drops incubated over different periods of time was increased $10 \times$ by introducing $0.2 \mathrm{M}$ sodium acetate buffer containing 3\% (w/V) sodium chloride. This facilitated sample collection and subsequent dilution in $0.07 \mathrm{M}$ potassium phosphate buffer, $\mathrm{pH} 6.24$, to obtain a final estimated concentration of $200-400 \mathrm{U} / \mathrm{mL}$. The enzymatic activity was measured following a colorimetric protocol that uses a suspension of Micrococcus lysodeikticus lyophilized cells as substrate. ${ }^{36}$ In short, $2.50 \mathrm{~mL}$ of M. lysodeikticus lyophilized cells $0.01 \%(\mathrm{w} / \mathrm{V}$ ) in $0.07 \mathrm{M}$ phosphate buffer, pH 6.24, was mixed with $0.10 \mathrm{~mL}$ of potassium phosphate buffer in a poly(methyl methacrylate) cuvette, and the $450 \mathrm{~nm}$ absorbance was read at $25^{\circ} \mathrm{C}$ in a Scansci Scanspec UV/vis spectrophotometer as a blank test. The same amount of $M$. lysodeikticus lyophilized cells was then mixed with $0.10 \mathrm{~mL}$ of the diluted HEWL drops. The absorbance decrease was monitored at $25^{\circ} \mathrm{C}$ for $300 \mathrm{~s}$, and the enzymatic activity was calculated from the slope of the linear portion of the progress curves. To check whether the molar concentration of lysozyme changed significantly with time, the $280 \mathrm{~nm}$ absorbance of the collected samples was measured at $25^{\circ} \mathrm{C}$ in a Scansci Scanspec UV/vis spectrophotometer.

\section{Results}

Movie S1 in Supporting Information reports the finding of nondissolving lysozyme crystals in a small drop accidentally spilled from a larger one containing $25 \mathrm{mg} / \mathrm{mL}$ HEWL in $0.2 \mathrm{M}$, pH 4.7 sodium acetate buffer, and 3\% (w/V) sodium chloride. Prepared and incubated at $37{ }^{\circ} \mathrm{C}$, the crystallization drops were initially undersaturated. The formation of HEWL crystals occurred normally in the two drops after decreasing the temperature down to $10{ }^{\circ} \mathrm{C}$ to create supersaturated conditions (not shown in the movie). The striking result was observed in the spilled drop (top of the movie frames) where complete dissolution of the crystals could not be achieved when the temperature was restored to $37^{\circ} \mathrm{C}$. The crystals in the interior of the larger drop followed the expected behavior and dissolved completely. In order to systematically study this phenomenon, crystallization drops of different volumes were subject to the successive temperature steps described in the Experimental Section and represented in Figure 1A. Dissolution phases purposely long were adopted to guarantee that partial dissolution was not determined by slow kinetics. We observed that HEWL crystals stopped dissolving after a variable number of cycles was completed and in a drop volume-dependent manner. Apparently, nondissolving crystals are produced at the end of fewer temperature cycles when smaller crystallization drops are used. In the example included in Figure 1A, total dissolution in $0.7 \mu \mathrm{L}$ drops could only be achieved at the end of the first three cycles. In the case of the stretched $0.4 \mu \mathrm{L}$ drop, the cessation of crystal dissolution occurred during the second dissolution cycle. In the round $0.4 \mu \mathrm{L}$ drop, dissolution stoppage was observed during the third cycle.

Despite the dispersion of behaviors observed from drop-to- drop, and from crystal-to-crystal within the same drop, a number of characteristic features were identified in the progress of the crystal sizes 
during growth and dissolution periods. Illustrating some of these features, Figure 1B,C shows that the variation of the equivalent diameter $E$ with time is approximately linear during the initial temperature steps. After a variable number of steps, curvilinear $\overline{L(t)}$ profiles are obtained indicating the occurrence of gradually slower phase transition processes. This tendency is sometimes culminated by the complete cessation of crystal dissolution and growth. As shown in Figure 1B, increasingly slow dissolution is not necessarily accompanied by increasingly slow crystal growth even when the dissolution process is practically ceased. Therefore, comparatively to dissolution, significant crystal growth retardation only occurs after additional temperature cycles are imposed. In the limit case reported in Figure 1C, the sixth growth/dissolution cycle shows no significant variation of the crystal size during each of the phases, meaning that changing the temperature from 37 to $10{ }^{\circ} \mathrm{C}$ and back to $37^{\circ} \mathrm{C}$ has apparently no effect on the crystal-solution equilibrium. Representing the results in Figure 1B,C in terms of the growth and dissolution rates (Figure 2) helps visualizing the two main conclusions drawn thus far: first, dissolution rates rapidly drop from cycle to cycle, especially in smaller drops; second, crystal growth rates also decrease, yet not so drastically and after more temperature cycles are completed. Figure $2 \mathrm{~A}, \mathrm{~B}$ includes examples of stopped dissolution during one of the $37{ }^{\circ} \mathrm{C}$ steps followed by restored dissolution at the beginning of the subsequent $37^{\circ} \mathrm{C}$ step (after an intermediate period of growth at $10^{\circ} \mathrm{C}$ ). On the contrary, crystal growth rates either remain approximately constant (Figure 2A) or steadily decrease (Figure 2B) with the succession of temperature steps. Consequently, a third observation is that crystal rebirth during consecutive cycles is likely to happen for the dissolution process but not for growth.

The insets in Figure 2A,B show that the size of the crystals at the end of the crystallization phases is clearly increased after the dissolution stoppages start to be observed, and that the number of crystals in the drops does not change significantly. As a result, the overall concentration of soluble HEWL is decreasing as the experiment is progressing and bigger crystals are produced - fourth conclusion. To check whether the integrity of the HEWL solution is affected in the crystallization drops, the glycoside hydrolase activity of lysozyme was periodically measured during the incubation of $0.7 \mu \mathrm{L}$ drops. Figure 3 shows a significant decrease of the catalytic activity with time, thus indicating that the native structure of a great fraction of

HEWL molecules becomes altered - fifth result. The effect of the volume of the drop on the rate of deactivation is not clear under the degree of uncertainty of these results. The measured values of absorbance in the inset of Figure 3 indicate that the loss of enzymatic activity of lysozyme is not accompanied bya significant variation of the molarconcentration.

\section{Discussion}

The succession of temperature cycles provoked an overall increase of the crystal sizes (fourth observation) and the consequent depletion of soluble HEWL during the microbatch experiments. This in turn can be used to explain the decreasing crystal growth rates (second observation) as the result of the lower supersaturation level $\sigma$ defined as $\sigma=\left(C-C^{*}\right) / C^{*}$, with $C$ being the protein concentration in solution and $C^{*}$ the protein solubility. Similarly, if nondissolving crystals are admittedly in a (pseudo) equilibrium stage, intermittent dissolution rates (third observation) can be explained by the HEWL concentration drop taking place during the intermediate period of growth at $10{ }^{\circ} \mathrm{C}$. When the temperature is increased back to $37^{\circ} \mathrm{C}$, the protein solution becomes undersaturated 
$\left(C^{*}>C\right)$ and dissolution is restored. However coherent this reasoning sounds, our first and more interesting question remains unanswered: why do HEWL crystals stop dissolving during the supposed dissolution step? This phenomenon has similarities with that of "crystal growth cessation" originally observed during lysozyme crystallization ${ }^{37}$ as the result of impurity poisoning of the crystal surfaces. ${ }^{34,37-39}$ It is not likely, however, that the purely diffusive step of crystal dissolution can be completely inactivated by minor concentrations of impurities at the same time that the propagation of surface steps during growth remains unaffected. We have also considered the important role of inhomogeneous concentration profiles along crystallization drops that have large surface area to volume ratios. In such cases, a great amount of soluble protein is adsorbed the liquid interface thus decreasing the bulk concentration in the equivalent amount. ${ }^{16,40,41}$ In vapor diffusion experiments, drop evaporation additionally increases the concentration of protein at the interfaces, where high local supersaturation levels tend to originate new nucleation events. ${ }^{42}$ Taken alone, the differences between interfacial and bulk conditions cannot explain why crystals positioned in a fixed point of the drop neither dissolve nor grow when subjected to temperatures between 37 and $10{ }^{\circ} \mathrm{C}$. Variations in the local concentrations may justify unexpected growth or dissolution behaviors but not their simultaneous occurrence. A possible solution to this problem is connected with the interfacial adsorption of HEWL while promoting important conformational changes 43 and ultimately protein denaturation. ${ }^{41}$ Because of their larger interfacial area per unit of volume, smaller crystallization drops also have higher fractions of adsorbed macromolecules undergoing structural rearrangements that compromise, sometimes irreversibly, the native biological function. We propose that the loss of catalytic activity of soluble HEWL is accompanied by a gain of thermodynamic activity as manifested by the peculiar evolution of the dissolution rates. The stepby-step explanation of this mechanism involves the interfacial adsorption of HEWL molecules, followed by their partial unfolding and the consequential phenomenon of solvation-out graphically illustrated in Figure 4 and previously identified during the aggregation of proteins into amyloid fibrils. $32,33,44$ The exposure to solvent of a high number of otherwise buried amino acids decreases the net availability of water molecules participating in the solvation equilibrium. Under such conditions, the protein molecules that are leaving the crystal lattice during dissolution find themselves surrounded by less water molecules than what would be expected in freshly prepared solutions. This originates the effective concentration of protein to increase, (ii) the enthalpy-change of solution to become less negative, ${ }^{45}$ and (iii) the dissolution process to become progressively slower until it eventually stops. The train of events leading to solvation-out effects and dissolution interruption is slightly different for crystal growth: in this case, only the macromolecules that keep their native folding are able to leave the solution phase and integrate the crystal lattice. The thermodynamic activity rise caused by denaturation and the reduced availability of water molecules are therefore counterbalanced by the decreased concentration of correctly folded protein molecules in solution. Owing to this additional depletion of crystallization-competent macromolecules, the driving force governing crystal growth is globally less affected by the conformational rearrangements than the driving force for dissolution. In conclusion, non- dissolving crystals are the result of the partial denaturation of soluble protein, which increases the overall thermodynamic activity of HEWL through solvation-out effects; growth cessation only occurs during subsequent temperature cycles after the concentration of active HEWL in solution is further depleted due to crystal growth and protein unfolding. 
Proof-of-Concept. Figure 5 presents a numerical demonstration in which Figure 5A,B pose the premises of the problem, while Figure 5C,D offers its possible solution. Inferred from the measured evolution of crystal size (Figure 1), crystal number (Figure 2) and enzymatic activity (Figure 3), Figure 5A shows representative values of the total concentration of HEWL (main panel) and of its active fraction over time (inset). In the considered example, the first dissolution stoppage occurs during the third cycle as this is the first instance where the initial protein concentration $(25 \mathrm{mg} / \mathrm{mL})$ is not reestablished after dissolution. During the fourth step at $37^{\circ} \mathrm{C}$, crystal dissolution is only partially restored since the magnitude of the concentration increase does not compensate the concentration drop that resulted from the previous growth phase. During the fifth and sixth cycles, there is no HEWL concentration increase indicating that no significant crystal dissolution occurs. By adopting an illustrative solubility value that coincides with the final protein concentration of the sixth growth step $\left(C^{*} 0=22.8 \mathrm{mg} / \mathrm{mL}\right.$ ), we are replicating the case previously represented in Figures $1 \mathrm{C}$ and $2 \mathrm{~B}$, for which crystal growth stoppage was observed at the end of the last cycle. Independently of the constant value above $25 \mathrm{mg} / \mathrm{mL}$ chosen as the solubility concentration at $37{ }^{\circ} \mathrm{C}$ (in the present case, $C_{3}^{\frac{1}{3} 7}=29.1 \mathrm{mg} / \mathrm{mL}$ ), the occurrence of dissolution stoppages is not possible to simulate without addressing additional considerations. The estimated evolution of crystal dissolution rates represented in Figure 5B illustrates how the finding of nondissolving crystals is against the predictions solely based on the protein concentration profile. Instead of decreasing the rates of dissolution, the progressively depleted protein solutions are supposed to accelerate the dissolution process.

As discussed above, when the driving forces for crystallization and dissolution are rather computed using thermodynamic activities, the simultaneous interruption of both types of phase transition becomes reasonable and elucidating - Figure 5C,D. The dissolution process, contingent as it is on the solvent availability, is determined by the total thermodynamic activity of the soluble protein $(a \mathrm{~T})$, whereas the crystallization step is determined by the thermodynamic activity of the protein molecules that keep their structural integrity (represented in Figure 5C by $a_{\mathrm{a}}$, with the subscript standing for active). While $a_{\mathrm{T}}$ increases as more protein molecules become unfolded (solvation-out effect), the value of $a_{\mathrm{a}}$ is subject to opposing influences of the solvation-out and protein inactivation effects. Accordingly, in the example given in Figure 5C, $a_{\mathrm{T}}$ is assumed to linearly increase with the fraction of unfolded molecules $\left(1-x_{\mathrm{a}}\right)$, whereas $a_{\mathrm{a}}$ is assumed to remain numerically equivalent to the HEWL concentration. This choice of activity coefficients harmonizes the experimentally inferred results of concentration depletion and catalytic activity loss in Figure $5 \mathrm{~A}$ with the measured evolutions of crystal growth and dissolution rates previously shown in Figure 2. Since the phase equilibrium is defined in relation to standard conditions, the effects caused by changes in the protein conformation do not affect the thermodynamic solubilities, which are assumed to be numerically equivalent to the concentration values adopted in Figure 5A: $a^{*} 0=C_{1}^{*} 0$ and $a_{3}^{*} 7=C_{3}^{*} 7$. The new scenario evidenced by Figure 5D confirms that null dissolution rates are possible to occur earlier than growth cessation, even if the protein concentration decreases from cycle-to-cycle. Besides explaining the apparently paradoxical first and second results, the solvation- out and protein inactivation effects are consistent with the observed dissolution rebirth after the dissolution stoppages (third result). Also in accordance with the experimental data, the oscillations in the values of $R_{\mathrm{D}}$ between cycles are more pronounced than the observed in the crystal growth rates, which steadily decrease toward complete cessation (Figure 5D).

Practical Implications. The thermodynamic hypothesis that consistently explains the discovery 
of nondissolving lysozyme crystals has additional implications besides contributing to the fundamental understanding of dissolution and growth. In the light of the new findings, the current biomolecular crystallization protocols can be further optimized in order to decrease the structural instability caused by interfacial phenomena. Difficult to perceive in minimal volumes of mother-liquor, protein adsorption in the liquid-air, liquid- oil, or liquid-solid interfaces not only decreases the effective bulk concentration as it leads to conformational rearrangements that deplete the solution of crystallizable macromolecules. This is not so important in the case of robust, easy-to-crystallize proteins for which local inhomogeneities and solvation-out effects might even promote new nucleation events. On the contrary, the self-organization of problematic proteins into crystals often require the chemical stabilization of the molecular structure, $5,17,18$ so that any source of additional instability might compromise the phase transition process. The interfacial adsorption step preceding the conformational modifications can be addressed using biophysical methods as an alternative to the more drastic biochemical methods. In this respect, the use of reporter dyes that fluoresce as the polypeptide is thermally unfolded can be used to measure the denaturation midpoint $\left(T_{\mathrm{m}}\right)$ and conveniently assess the conditions of buffer, $\mathrm{pH}$, ionic strength, and additives that stabilize the macromolecular structure. ${ }^{46-48}$ Precipitants such as 2-methyl-2,4-pentanediol (MPD) that bind to exposed hydrophobic residues should be chosen to promote the preferential hydration and stabilization of the protein. ${ }^{49}$ Besides their solubility-decreasing and cryoprotectant properties, the effectiveness of MPD, poly- ethylene glycol (PEG), ${ }^{50,51}$ and glycerol ${ }^{52}$ as crystallization agents cannot be dissociated from their protective effects against noncovalent protein modifications. The same principle is applicable to the development of protein-stabilizing ionic liquids ${ }^{53}$ and efficient polymeric hydrogels able to create convection-free and protein-friendly crystallization environments. ${ }^{13,54,55}$ The new perspective provided by our results not only includes some of the known success stories in macro- molecular crystallization, but also contributes to highlight sample stability as a key factor to explain, e.g., why the largest protein crystals are observed to develop during the first few hours of the assay ${ }^{56}$ or why the vast majority of structures deposited by the Structural Genomics Consortium, Oxford, were determined from crystals produced within the first days of incubation. ${ }^{57}$ In agreement with recent recommendations, 2,56 the tendency to use increasingly smaller drop volumes should be looked with caution since, as shown here, high surface area to volume ratios may decisively accelerate protein adsorption and unfolding. Sample miniaturization can be reconciled with controlled interfacial phenomena by revisiting the type of construction materials, surface treatments, and cover oils adopted in vapor-diffusion, liquid-liquid diffusion, and microbatch methods. For example, we argue that the adsorption and unfolding of protein molecules at the solid- liquid interface is an important variable to be introduced in the debate over the most adequate materials to build microfluidic devices for protein crystallization. ${ }^{58}$

\section{Conclusions}

Nondissolving HEWL crystals are the result of the increased thermodynamic activity of the soluble protein under conditions of widespread interfacial adsorption and progressive protein unfolding. Having higher surface area to volume ratios, small solution drops promote the interfacial adsorption of protein molecules and their consequential denaturation. The exposure to solvent of buried amino 
acids is thought to decrease the number of water molecules available to participate in the dissolution process - solvation-out effect. Conformational in- stability is reflected in the measured loss of enzymatic activity of HEWL with time. The effective concentration of crystallizable macromolecules is subject to positive solvation-out effects and to negative protein inactivation effects. Hard-to-crystallize proteins tend to be more influenced by the latter effect which justifies further optimization of the crystallization protocols regarding the control of interfacial phenomena (particularly when miniaturized volumes are employed). This can be achieved by selecting the solution environment (buffer, $\mathrm{pH}$, ionic strength, additives and hydrogels) and interfaces (liquid- air, liquid-oil, and liquid-solid) that minimize interfacial protein unfolding.

\section{Associated content}

\section{Supporting Information}

The Supporting Information is available free of charge on the ACS Publications website at DOI: 10.1021/acs.cgd.6b00334.

Movie S1: Observation of crystal dissolution stoppage in the smaller crystallization drop. The 25 $\mathrm{mg} / \mathrm{mL}$ HEWL drops containing 3\% (w/V) sodium chloride, $\mathrm{pH} 4.7$ are undersaturated at $37{ }^{\circ} \mathrm{C}$. The total duration of the movie is approximately $750 \mathrm{~min}$ (AVI)

\section{Acknowledgments}

This work was financially supported by Project UID/EQU/ 00511/2013-LEPABE (Laboratory for Process Engineering, Environment, Biotechnology and Energy - EQU/00511) by FEDER funds through Programa Operacional Competitividade e Internacionalizaç⿻ COMPETE2020 and by national funds through FCT - Fundaçpara a Ciênia e a Tecnologia. C.F. gratefully acknowledges Grant No. SFRH/BD/74174/2010 FCT, Portugal.

\section{References}

1. Giege,R. FEBS J. 2013, 280, 6456.

2. Luft, J. R.; Newman, J.; Snell, E. H. Acta Crystallogr., Sect. F: Struct. Biol. Commun. 2014, 70,835 .

3. McPherson, A.; Gavira, J. A. Acta Crystallogr., Sect. F: Struct. Biol. Commun. 2014, 70, 2.

4. Buschmann, S.; Richers, S.; Ermler, U.; Michel, H. Protein Sci. 2014, 23, 411.

5. Bukowska, M. A.; Grütter, M. G. Curr. Opin. Struct. Biol. 2013, 23, 409.

6. Yonath, A. Curr. Opin. Struct. Biol. 2011, 21,622.

7. Giege,R. Cryst. Growth Des. 2013,13, 405.

8. Shaw Stewart, P.; Mueller-Dieckmann, J. Acta Crystallogr., Sect. F: Struct. Biol. Commun. 2014, 70,686 .

9. Zhu, Y.; Fang, Q. Anal. Chim. Acta 2013, 787, 24.

10. Garcia-Caballero, A.; Gavira, J. A.; Pineda-Molina, E.; Chayen, N. E.; Govada, L.; Khurshid, S.; Saridakis, E.; Boudjemline, A.; Swann, M. J.; Shaw Stewart, P. Cryst. Growth Des. 2011, 11, 
2112-2121.

11. Khurshid, S.; Saridakis, E.; Govada, L.; Chayen, N. E. Nat. Protoc. 2014, 9, 1621.

12. D’Arcy, A.; Bergfors, T.; Cowan-Jacob, S. W.; Marsh, M. Acta Crystallogr., Sect. F: Struct. Biol. Commun. 2014, 70, 1117.

13. Gavira, J. A.; Cera-Manjarres, A.; Ortiz, K.; Mendez, J.; Jimenez- Torres, J. A.; Patiño-Lopez, L. D.; Torres-Lugo, M. Cryst. Growth Des. 2014, 14, 3239.

14. Crespo, R.; Martins, P. M.; Gales, L.; Rocha, F.; Damas, A. M. J. Appl. Crystallogr. 2010, 43, 1419.

15. Ferreira, C.; Crespo, R.; Martins, P. M.; Gales, L.; Rocha, F.; Damas, A. M. CrystEngComm $2011,13,3051$.

16. Martins, P.; Pessoa, J.; Sarkany, Z.; Rocha, F.; Damas, A. Cryst. Growth Des. 2008, 8, 4233.

17. Rosenbaum, D. M.; Cherezov, V.; Hanson, M. A.; Rasmussen, S. G.; Thian, F. S.; Kobilka, T. S.; Choi, H.-J.; Yao, X.-J.; Weis, W. I.; Stevens, R. C.; Kobilka, B. K. Science 2007, 318, 1266.

18. Derewenda, Z. S. Structure 2004, 12, 529.

19. Dale, G. E.; Oefner, C.; D’Arcy, A. J. Struct. Biol. 2003, 142, 88.

20. Deller, M. C.; Kong, L.; Rupp, B. Acta Crystallogr., Sect. F: Struct. Biol. Commun. 2016, 72, 72.

21. Bolanos-Garcia, V. M.; Chayen, N. E. Prog. Biophys. Mol. Biol. 2009, 101, 3.

22. Forsythe, E. L.; Judge, R. A.; Pusey, M. L. J. Chem. Eng. Data 1999, 44, 637.

23. Galkin, O.; Vekilov, P. G. J. Cryst. Growth 2001, 232, 63.

24. Aldabaibeh, N.; Jones, M. J.; Myerson, A. S.; Ulrich, J. Cryst. Growth Des. 2009, 9, 3313.

25. Liu, Y.; Wang, X.; Ching, C. B. Cryst. Growth Des. 2010, 10, 548.

26. Oswald, R.; Ulrich, J. Cryst. Growth Des. 2015, 15, 4556.

27. Basu, S. K.; Govardhan, C. P.; Jung, C. W.; Margolin, A. L. Expert Opin. Biol. Ther. 2004, 4,301 .

28. Ulrich, J.; Pietzsch, M. Cryst. Res. Technol. 2015, 50, 560.

29. Markov, I. V. Crystal Growth for Beginners: Fundamentals of Nucleation, Crystal Growth and Epitaxy; World Scientific, Singapore, 2003.

30. Dokoumetzidis, A.; Macheras, P. Int. J. Pharm. (Amsterdam, Neth.) 2006, 321,1.

31. Ellis, R. J. Trends Biochem. Sci. 2001, 26, 597.

32. Crespo, R.; Villar-Alvarez, E.; Taboada, P.; Rocha, F. A.; Damas, A. M.; Martins, P. M. J. Biol. Chem. 2016, 291, 2018.

33. Crespo, R.; Villar-Alvarez, E.; Taboada, P.; Rocha, F. A.; Damas,

A. M.; Martins, P. M. The availability of soluble amyloidogenic protein is decreased in the presence of insoluble off-pathway aggregates, 2016.

34. Ferreira, C.; Rocha, F. A.; Damas, A. M.; Martins, P. M. J. Cryst. Growth 2013, 368, 47.

35. Ferreira, C.; Rocha, F. A.; Damas, A. M.; Martins, P. M. Cryst. Growth Des. 2012, 12, 40.

36. Davies, R.; Neuberger, A.; Wilson, B. Biochim. Biophys. Acta, Enzymol. 1969, 178, 294.

37. Durbin, S. D.; Feher, G. J. Cryst. Growth 1986, 76, 583.

38. Sáiahez-Pastor, N.; Pina, C. M.; Fernádez-Díaz, L.; Astilleros, J. M. Surf. Sci. 2006, 600, 1369.

39. Martins, P. M.; Rocha, F.; Damas, A. M.; Rein, P. CrystEngComm 2011, 13, 1103.

40. Miller, R.; Fainerman, V.; Makievski, A.; Leser, M.; Michel, M.; Aksenenko, E. Colloids Surf., 
B 2004, 36, 123.

41. Alahverdjieva, V.; Grigoriev, D. O.; Ferri, J.; Fainerman, V.; Aksenenko, E.; Leser, M.; Michel, M.; Miller, R. Colloids Surf., A 2008, 323, 167.

42. García-Ruiz, J.; Otaĺora, F.; García-Caballero, A. Acta Crystallogr., Sect. F: Struct. Biol. Commun. 2016, 72, 96.

43. Kubiak-Ossowska, K.; Mulheran, P. A. Langmuir 2010, 26, 15954.

44. Crespo, R.; Rocha, F. A.; Damas, A. M.; Martins, P. M. J. Biol. Chem. 2012, 287, 30585.

45. Collins, K. D. Methods 2004, 34, 300.

46. Ericsson, U. B.; Hallberg, B. M.; DeTitta, G. T.; Dekker, N.; Nordlund, P. Anal. Biochem. 2006, 357, 289.

47. Ristic, M.; Rosa, N.; Seabrook, S. A.; Newman, J. Acta Crystallogr., Sect. F: Struct. Biol. Commun. 2015, 71, 1359.

48. Reinhard, L.; Mayerhofer, H.; Geerlof, A.; Mueller-Dieckmann, J.; Weiss, M. S. Acta Crystallogr., Sect. F: Struct. Biol. Cryst. Commun. 2013, 69, 209.

49. Anand, K.; Pal, D.; Hilgenfeld, R. Acta Crystallogr., Sect. D: Biol. Crystallogr. 2002, 58, 1722.

50. Radaev, S.; Sun, P. D. J. Appl. Crystallogr. 2002, 35, 674.

51. Vera, L.; Stura, E. A. Cryst. Growth Des. 2014, 14, 427.

52. Vera, L.; Czarny, B.; Georgiadis, D.; Dive, V.; Stura, E. A. Cryst. Growth Des. 2011, 11, 2755.

53. Pusey, M. L.; Paley, M. S.; Turner, M. B.; Rogers, R. D. Cryst. Growth Des. 2007, 7, 787.

54.Lorber, B.; Sauter, C.; Thébald-Dietrich, A.; Moreno, A.; Schellenberger, P.; Robert, M.-

C.; Capelle, B.; Sanglier, S.; Potier, N.; Giege,R. Prog. Biophys. Mol. Biol. 2009, 101, 13.

55. Gavira, J. A.; Van Driessche, A. E.; Garcia-Ruiz, J.-M. Cryst. Growth Des. 2013, 13, 2522.

56. McPherson, A.; Cudney, B. Acta Crystallogr., Sect. F: Struct. Biol. Commun. 2014, 70, 1445.

57.Ng,J.T.;Dekker, C.; Reardon, P.; vonDelft,F.Acta Crystallogr., Sect.D:Struct. Biol.2016, 72, 224.

58.Dhouib, K.; Khan Malek, C.; Pfleging, W.; Gauthier-Manuel, B.; Duffait, R.; Thuillier, G.; Ferrigno, R.; Jacquamet, L.; Ohana, J.; Ferrer, J.-L.; Theobald-Dietrich, A.; Giege, R.; Lorber, B.; Sauter, C. Lab Chip 2009, 9, 1412. 


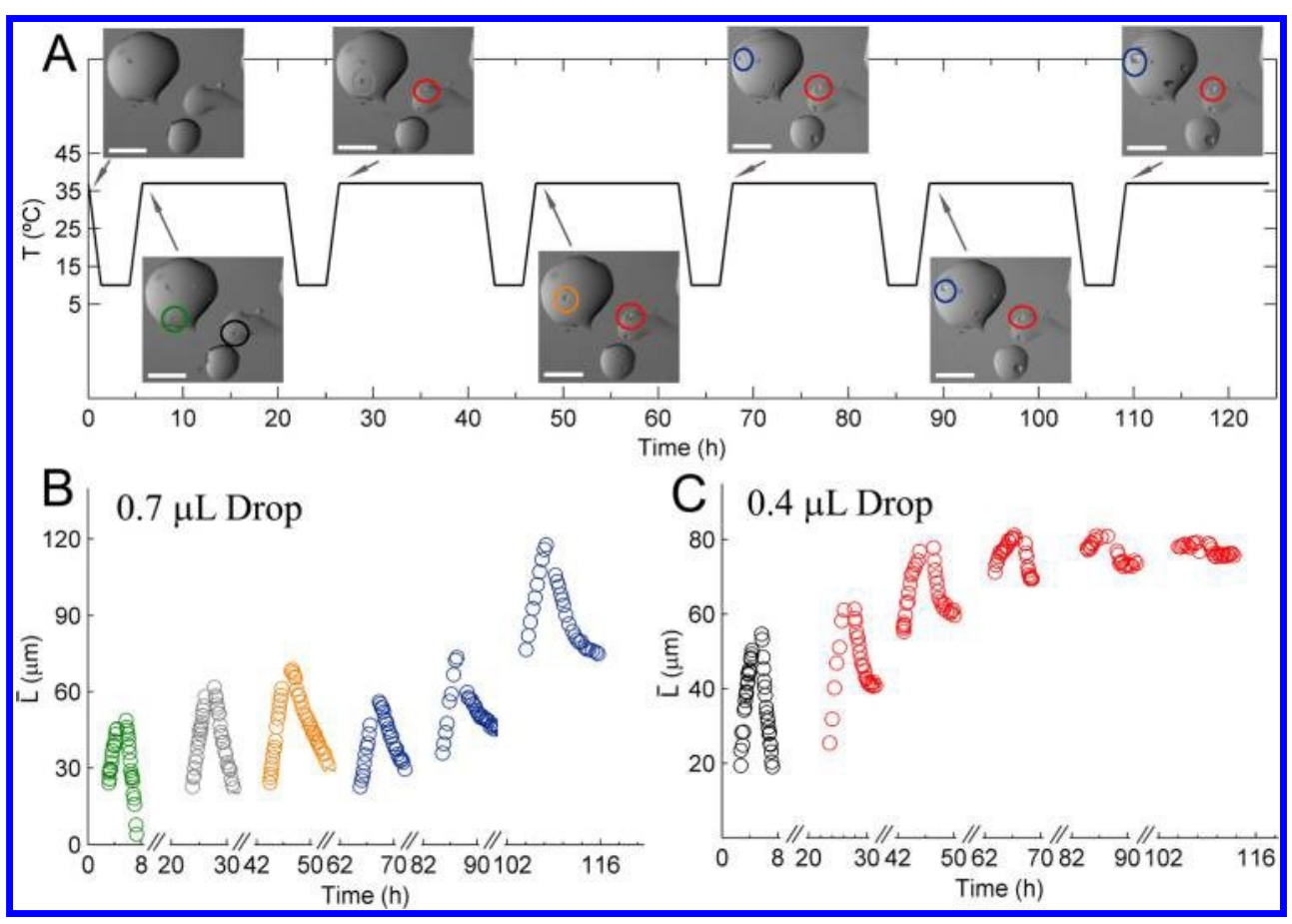

Figure 1. Observation of crystal formation and dissolution in sub-microliter $25 \mathrm{mg} / \mathrm{mL}$ HEWL drops containing 3\% (w/V) sodium chloride, $\mathrm{pH} 4.7$, placed in a jacketed growth cell and covered with paraffin oil. (A) Temperature program imposed to promote crystallization (at $10{ }^{\circ} \mathrm{C}$ ) and crystal dissolution (at $37^{\circ} \mathrm{C}$ ). Succession of images: monitoring the occurrence of HEWL crystals within one $0.7 \mu \mathrm{L}$ drop and two $0.4 \mu \mathrm{L}$ drops before each dissolution period. Selected crystals are identified by different colored circles. The scale bar is $1.0 \mathrm{~mm}$. The arrows indicate the instant the images were taken. (B and C) The equivalent diameter Lof the selected crystals is followed over time. Different colors mean different crystals as in (A).

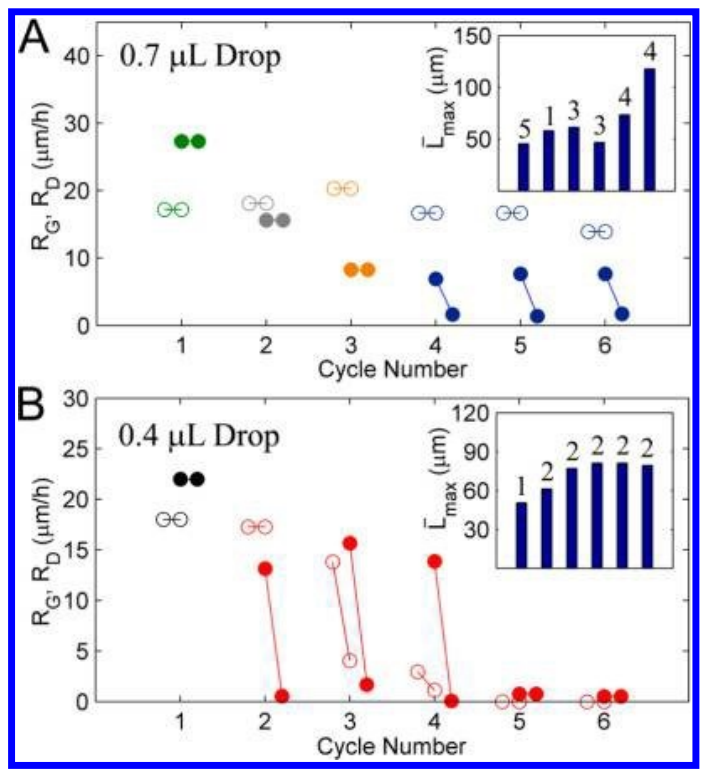

Figure 2. Variation of the crystal growth rate $\left(R_{\mathrm{G}}\right.$, open symbols) and dissolution rate $\left(R_{\mathrm{D}}\right.$, solid symbols) over a period of $3 \mathrm{~h}$ during each temperature cycle. Data computed from the progress of 
crystal sizes represented in (A) Figure 1B and (B) Figure 1C. Insets: Equivalent diameters Lof the selected crystals at the end of the crystallization periods. The values on top of the vertical bars indicate the number of crystals simultaneously present in the selected drop.

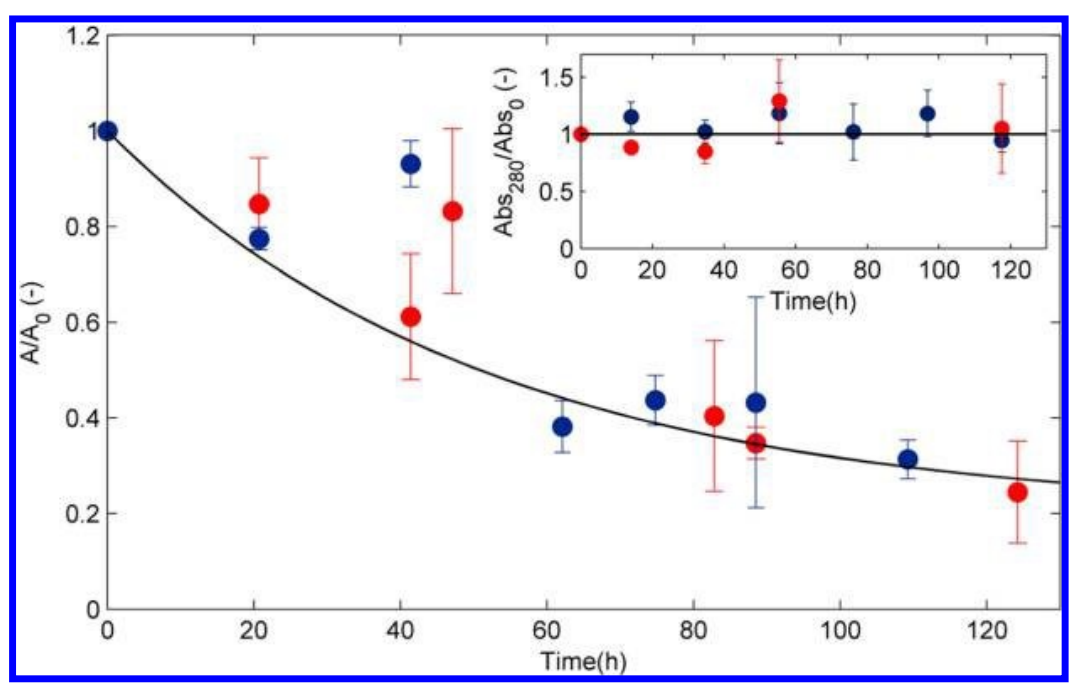

Figure 3. Loss of enzymatic activity of lysozyme in $0.7 \mu \mathrm{L}$ (blue) and $0.4 \mu \mathrm{L}$ (red) drops subjected to the experimental conditions and temperature program previously described in Figure 1. The enzymatic activity was measured using Micrococcus lysodeikticus lyophilized cells as substrate. ${ }^{36}$ Symbols and error bars: average values and standard deviations of replicate measurements of the enzymatic activity normalized over the initial average value $\left(A / A_{0}\right)$. All samples were collected at 37 ${ }^{\circ} \mathrm{C}$. Line: exponential decay function used as a guide to the eye. Inset: average values and standard deviations of replicate measurements of the $280 \mathrm{~nm}$ absorbance of the $0.7 \mu \mathrm{L}$ (blue) and $0.4 \mu \mathrm{L}$ (red) drops normalized over the initial average value (Abs280/Abs0); apparently constant Abs280/Abs0 values indicate that the molar concentration of lysozyme did not change significantly over time.

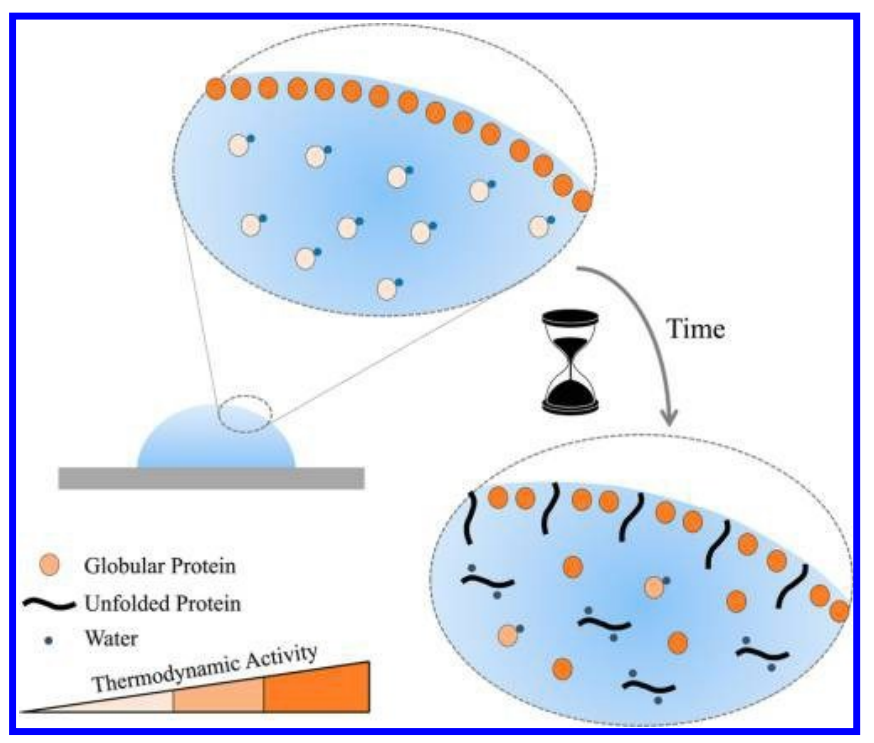

Figure 4. Interfacial adsorption of protein molecules at the liquid-air interface promotes the 
progressive unfolding of globular proteins, which in turn decreases the net availability of water molecules. The increased thermodynamic activity of soluble protein can explain the occurrence of the dissolution stoppages. During crystallization, this effect is counterbalanced by the decreased amount of correctly folded macromolecules.

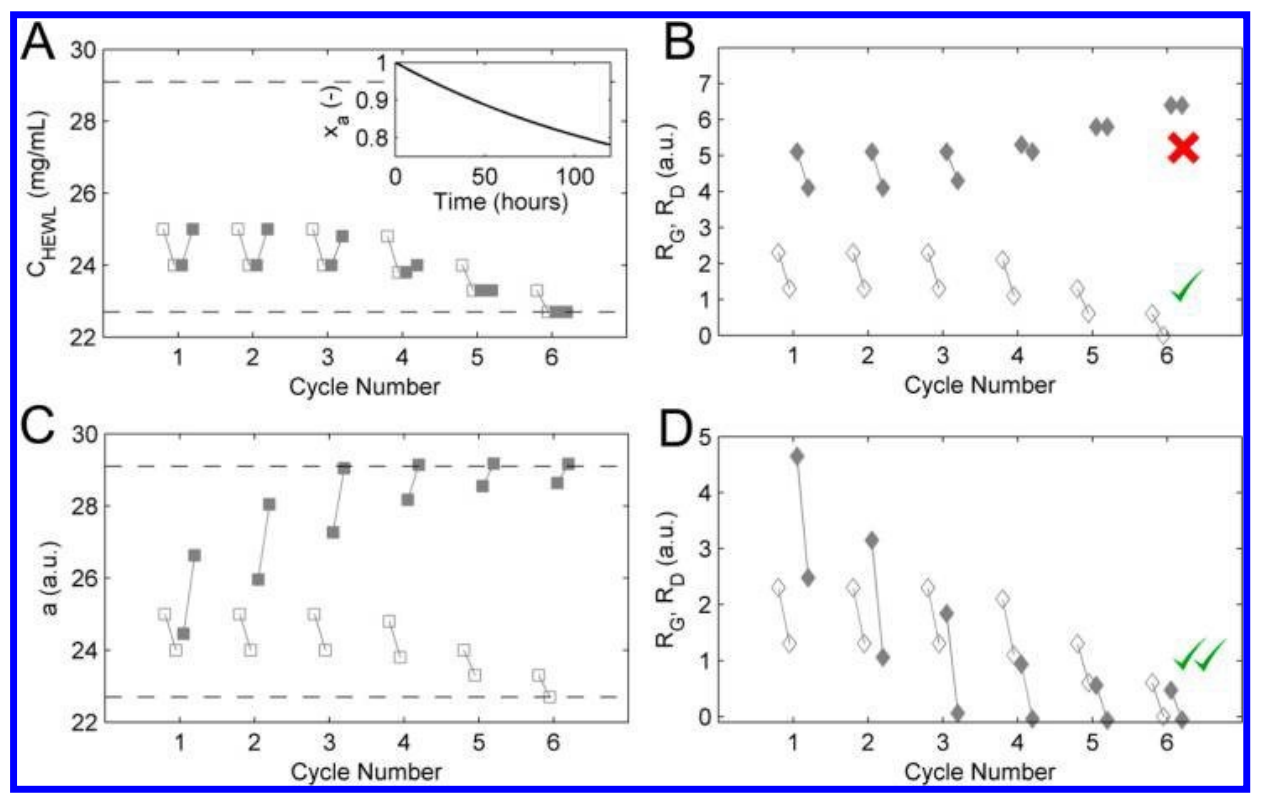

Figure 5. Crystal dissolution stoppages succeeded by crystal growth stoppages are explained by variations in the thermodynamic activity of soluble HEWL upon protein unfolding-proof-ofconcept. (A) Numerical example of the HEWL concentration profile during the growth periods (open symbols) and dissolution periods (solid symbols) computed from the measured evolution of crystal size (Figure 1) and crystal number (Figure 2) in the sub-microliter drops. Dashed lines: exemplifying values of the HEWL solubility during the growth ( $C_{10}^{*}$ ) and the dissolution ( $\left.\mathrm{C}_{37}^{*}\right)$ periods. Inset: exponential decrease of the fraction HEWL molecules preserving their native structure $\left(x_{\mathrm{a}}\right)$ as a function of time $(t)$; progress curve computed as $x_{\mathrm{a}}=\left(0.6+0.4 \mathrm{e}^{-6 \cdot 61 \times 10^{-3} t}\right)$. (B) Crystal growth rates $(R$, open symbols) and dissolution rates $(R$, solid symbols) at the beginning and end of each cycle computed assuming direct proportionality between $R_{G}$ and $\left(C-C_{1}^{*}\right)$, and between $R_{D}$ and $\left(C_{37}-C\right)$; only crystal growth stoppage is admissible in this scenario. (C) The concentration profiles in (A) are converted into thermodynamic activity profiles of active HEWL $\left(a_{\mathrm{a}}\right)$ and total soluble HE $\left(a_{\mathrm{T}}\right)$ in arbitrary units; the value of $a_{\mathrm{T}}$ is assumed to linearly increase with the amount of unfolded protein: $a_{\mathrm{T}}=C\left(1+1.2\left(1-x_{\mathrm{a}}\right)\right)$; this effect is counterbalanced in the value of $a_{\mathrm{a}}$ by the decreased concentration of active HEWL, so that $a_{\mathrm{a}}$ is assumed to numerically correspond to $\sim C$. Dashed lines: exemplifying values of the equilibrium thermodynamic activity of HEWL at $10^{\circ} \mathrm{C}\left(a^{*} 0\right)$ and at $37^{\circ} \mathrm{C}$ ( $a_{3}^{\frac{*}{3}} 7$ ). (D) Values of $R_{\mathrm{G}}$ (open symbols) and $R_{\mathrm{D}}$ (solid symbols) at the beginning and end of each cycle computed assuming direct proportionality between $R_{\mathrm{G}}$ and $\left(a_{\mathrm{a}}-a_{1}^{*}\right)$ ), and between $R_{\mathrm{D}}$ and ( $a^{*}$ - a37); dissolution stoppages (at the end of cycles third to sixth) and crystal growth stoppage (sixth cycle) are here admissible; dissolution rebirth is also possible as during the growth-dissolution transition from the third to the fourth cycle. 